\title{
La participation des fellahs à l'agriculture irriguée dans l'Egypte contemporaine
}

\author{
par Thierry Ruf \\ ORSTOM
}

\section{I 圆 INTRODUCTION}

L'agriculture égyptienne est l'une des plus intensives et productives du monde actuel. Le système de culture-type est une succession de six cultures sur un cycle de trois ans, trèfle et coton la première année, trèfle et maïs la seconde année, blé et maïs la troisième année. L'exploitation agricole moyenne est d'un demi-hectare. La productivité est élevée, équivalente à 10 tonnes de grains par hectare et par an. Ces systèmes valorisent au maximum le travail agricole et l'eau, mais intègre aussi des outils spécialisés dans le travail du sol et l'exhaure. La mécanisation de la préparation des terres vise à accélérer la transition entre deux cultures successives Les pompes diesel se sont substituées partiellement ou totalement aux norias mues par des animaux, afin d'exploiter le maximum du volume délivré par le service des irrigations pendant six jours sur dix-huit.

La densité actuelle est de plus de 2000 habitants au kilomètre carré agricole utile. Il y a deux siècles, la population était voisine de deux millions d'habitants, contre 65 millions aujourd'hui. En étudiant la transition entre le système millénaire de culture de décrue qui régnait encore en 1830 et le système de cultures irriguées de la fin du $\mathrm{Xx}^{\mathrm{e}}$ siècle, on constate que les innovations précèdent la croissance démographique.

\section{The fellah's participation to the irrigated agriculture in the contemporaneous Egypt}

The landscapes of the Nile's valley and delta have been completely transformed during the last two centuries: the controled submersion has gradually disappeared towards the perennat irrigation. The process is prior to the High Dam of Assouan, which comes to conclude the new water management set of the Nile; it begun with the Saint-Simoniens in the 1830 -1840s and achieved in the $1960 \mathrm{~s}$ with water storcage, and in the 1980-1090; as for dranage adjustment.

During the $19 t h$ century, the interest of the State falls on a cash crop, cotton with long fibers. It takes place in the cropping pattern where also figures wheat, clover (bersin) and corn (r Turkish wheat $>$ ). As a tropical crop, cotton cannot support the cold of the winter In order to be chltivated it has to be sown in Februa y-March and harvested in Angust at the time when the Nile is in flood. The landscape clanges: the State tries to delay and lo avoid the flood. It has to bring the water artificially during he low water level of the river. The saktid becomes the instrument of integration in the scale of the fellahs in a context of new hydraulic conditions, land tenure reform and controled agriculture markets.

The High Dam of Assouan avoids the major nisk of villages and crops being destroyed. The delay taken in programs of drainage indices processes of salinisation, while the ubanisation left lands to the so limited agricultural area. 


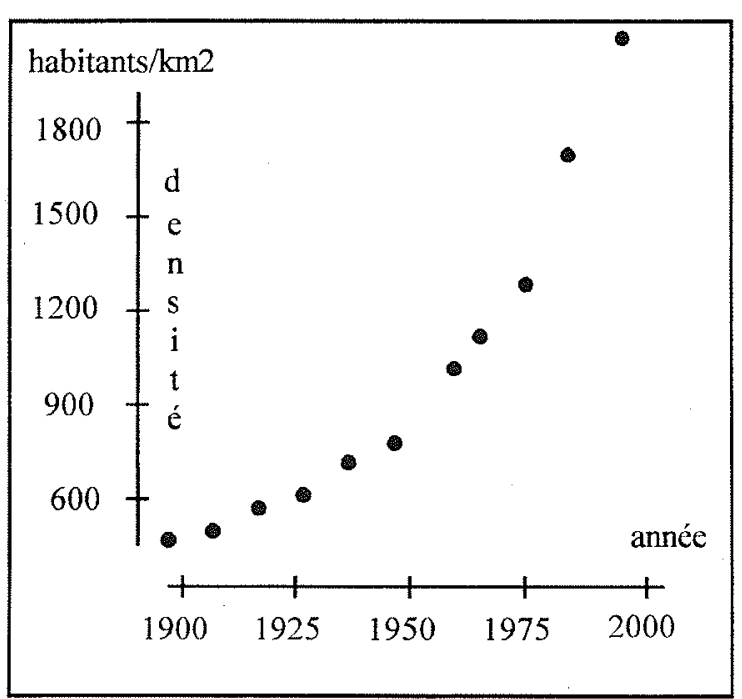

1. Evolution de la densité de la population totale par rapport à la superficie agricole.

\section{II 圆 L'ÉTAT ET LA PAYSANNERIE AVANT LA CONVERSION DES BASSINS DE DÉCRUE À L'IRRIGATION PÉRENNE}

Les paysages de la vallée et du delta du Nil ont été complètement transformés au cours des deux derniers siècles: la submersion contrôlée a progressivement disparu au profit de l'irrigation pérenne. Le processus est bien antérieur au Haut Barrage d'Assouan, ouvrage venant conclure l'ensemble du réaménagement du Nil commencé avec les Saint Simoniens dans les années 1830-1840.

La crue du Nil était prévisible mais incertaine quant à sa force, à sa durée. L'étiage lui-même n'était parfois pas assez vite atteint. La crue a été maîtrisée avec des séries de bassins récepteurs le long de la vallée et des branches du Delta. La réussite de la submersion n'était pas garantie si les bassins supérieurs retenaient l'eau ou la lâchaient trop vite. Les digues mal entretenues pouvaient céder sous la pression de l'eau.

Le droit foncier sur la terre inondée differe totalement du droit de l'eau sur une terre asséchée. Dans le premier cas, la culture de décrue n'est réalisable qu'en vertu d'une concession renouvelée chaque année, en fonction de la crue, par une autorité régionale. Dans le second cas, l'eau est au centre de la vie sociale et économique d'un groupe restreint, fondateur ou héritier de fondateurs du système de captation et de distribution. Enfin, dans I'hydraulique ancienne de l'Egypte, le plus difficile n'est probablement pas de recevoir une crue, mais de coordonner la vidange des séries de bassins de 5000 hectares avec la mise en culture des terres progressivement exondées.

La crue empêchait la pratique de cultures permanentes, sauf sur les bourrelets de berge ou sur les bords de la vallée restant toujours hors de portées des plus hautes eaux. Quand le Nil était à l'étiage, on irriguait ces zones restreintes avec des sakkias (roues avec chaine de godets).

\section{LA STRATÉGIE COTONNIÈRE DE

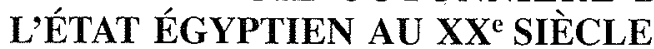

La première révolution agricole est d'abord institutionnelle et politique avec la reprise en main de l'Etat par un despote moderniste, Mohammed Ali (1805-1848). C'est lui qui éli- mine l'ancienne caste militaire mamelouk en 1811 et provoque l'une des premières véritables réformes agraires contemporaines. Il perçoit l'avantage économique qu'il peut tirer de la découverte de variétés de cotonnier à longues soies (Jumel, 1820). Il impose un renversement total de la stratégie Etatique de gestion des crues du Nil (juillet-septembre) en ne s'intéressant plus qu'à l'étiage (février-juin) pour pouvoir étendre la culture du cotonnier irriguée (18201840). Il oblige les paysans à cultiver le coton sur les anciennes terres des mamelouks, et finance les norias indispensables pour aller chercher l'eau au fond des canaux mal alimentés en période de basses eaux.

Placés dans ce nouveau contexte, les fellahs devenus par force cultivateurs de coton avant d'être céréaliculteurs vont être de plus en plus soumis à une exploitation excessive de leur travail. Cependant, après 1860 , les effets de la guerre de sécession aux Etats-Unis d'Amérique entraînent un enrichissement rapide des fellahs et de l'Etat. Economiquement éphémère, le boom cotonnier a des conséquences sociales durables: les fellahs se trouvent désormais petits propriétaires fonciers, mieux armés pour produire du coton en masse que les grands propriétaires qui manquent de main d'œuvre. En revanche, l'endettement public aboutit à la faillite de l'Etat et à la mise sous tutelle de l'Egypte par les puissances occidentales.

\section{LE LIEN ENTRE L'AMÉNAGEMENT ETATIQUE ET LA PAYSANNERIE}

A la fin du $\mathrm{XIX}^{\mathfrak{e}}$ siècle, le débit d'étiage devient limitant. On commence à construire un premier barrage de stockage à Assouan (1898-1902) et des barrages d'élévation des plans d'eau pour faciliter l'admission des basses eaux dans les canaux. Le phénomène de salinisation des terres agricoles irriguées apparaît en Egypte à cette période. Les travaux des agronomes sur ses effets de la remontée de la nappe phréatique sur le cotonnier, au début $\mathrm{du} x \mathrm{x}^{\mathrm{e}}$ siècle, induisent un débat difficile entre agronomes et hydrauliciens sur les remèdes à apporter. Le projet des hydrauliciens était de relever tous les plans d'eau et de supprimer l'exhaure par les sakkias. Les agronomes voulaient maintenir l'alternance de biefs en eau et de biefs vides dans les canaux afin de limiter la remontée de la nappe phréatique. En définitive, l'Etat égyptien décide de maintenir l'alternance de mise en eau des canaux (6 jours pleins, 12 jours vides) pour éviter la surirrigation permanente. En outre, la mise en place du réseau de drainage par grands fossés publics fut décidée dans les années 1910-1920. Et les sakkias vont rester indispensables pour assurer l'arrosage de toutes les cultures.

Démarré au XIX ${ }^{e}$ siècle dans le delta du Nil et certains secteurs de la vallée, le programme de conversion des bassins se poursuit au $\mathrm{XX}^{\mathrm{e}}$ siècle. Au moment de la mise en service du Haut Barrage d'Assouan (1964), $80 \%$ des anciens bassins avaient déjà été adaptés. Le Haut Barrage lève le risque majeur de destruction des villages et des cultures.

Dans cette période qui voit l'Egypte trouver son indépendance, le cotonnier reste la culture gouvernementale, toujours obligatoire par grandes soles, indépendamment du fractionnement de la propriété foncière, mais tributaire du maillage hydraulique des réseaux, au fur et à mesure des perfectionnements hydrauliques pour la mâtrise des plans d'eau.

Localement, la noria ou sakkia persiste jusque dans les années 1970-1980, comme interface entre les changements de gestion décrétés par l'Etat et les systèmes de production élaborés par les petits propriétaires. Elle est à la base de l'association entre l'agriculture et l'élevage au sein même de l'exploitation paysanne. La principale innovation consiste à accroittre la production fourragère dans un système de fortes 
contraintes: l'Etat interdit toutes les cultures fourragères d'été pour limiter l'extension des maladies parasitaires du cotonnier. Le trèfle devient la culture d'hiver principale et même le blé est produit pour sa paille. La base de l'alimentation paysanne passe du blé (culture d'hiver) au maïs (culture d'été) que l'on pouvait difficilement cultiver lorsque les crues n'étaient pas maîtrisées.

\section{LA LUTTE DES FELLAHS POUR ÉCHAPPER À LA PAUPÉRISATION}

L'un des facteurs qui a contribué à l'essor d'une économie paysanne est la lutte des familles pour rester au-dessus d'un seuil de paupérisation. En terme de superficie cultivée par une famille nucléaire, le seuil est passé de 2,1 hectares au début du XIX ${ }^{\mathrm{e}}$ siècle en culture de décrue sans outillage, à 0,4 hectare en culture irriguée intensive et en partie mécanisée. En dessous de ce seuil, les familles ne produisent plus leur base d'alimentation. Au-dessus, elles maintiennent un équilibre relatif entre trois objectifs : se nourrir, accéder aux échanges monétaires (indispensables pour l'exhaure) et conserver une ou plusieurs têtes de bétail (tabl. $l$ ). L'élevage est la clé de voûte du système de production par les trois fonctions essentielles: la force de traction, l'épargne sur pied, et la fertilisation organique. Cette dernière fonction est essentielle, elle conduit les fellahs à gérer un continuel transfert de fourrages des champs au village et des matières organiques du village vers les champs.

Cependant, de graves problèmes subsistent ou apparaissent avec cette intensification de l'agriculture et l'explosion démographique. Ainsi, le retard pris dans les programmes de drainage induit des processus de salinisation tandis que l'urbanisation met en péril les terres agricoles. Pour perdre le moins de temps possible, la mécanisation de la préparation des terres vise à accélérer la transition entre deux cultures ou trois cultures successives. Les pompes diesel se sont substituées aux sakkias mues par des animaux, afin d'exploiter au mieux le volume délivré par le service des irrigations pendant six jours sur dix-huit, ce qui perturbe beaucoup le cycle de remplissage des segments de canaux. L'usage

Tableau 1. - Typologie simplifiée des exploitations paysannes dans le delta du Nil à la fin du $x x^{e}$ siècle.

\begin{tabular}{|c|c|c|c|c|c|c|}
\hline $\begin{array}{c}\text { Types } \\
\text { indicateurs }\end{array}$ & \multicolumn{2}{|c|}{ Groupe 1} & \multicolumn{2}{|c|}{ Groupe 2} & Groupe 3 & Groupe 4 \\
\hline Superficie & \multicolumn{2}{|c|}{$\begin{array}{l}<1 \text { feddan } \\
(<0,42 \text { ha })\end{array}$} & \multicolumn{2}{|c|}{$\begin{array}{c}1 \text { à }<5 \text { feddans } \\
\text { (de } 0,42 \text { à } 2,1 \text { ha) }\end{array}$} & $\begin{array}{l}5 \text { à }<20 \text { feddans } \\
(2,1 \text { à } 8,4 \text { ha })\end{array}$ & $\begin{array}{c}>20 \text { feddans } \\
(>8,4 \text { ha })\end{array}$ \\
\hline $\begin{array}{l}\text { Bloc } \\
\text { culture }\end{array}$ & \multicolumn{2}{|c|}{1 ou plusieurs } & \multicolumn{2}{|c|}{1 ou plusieurs } & plusieurs & plusieurs \\
\hline $\begin{array}{l}\text { Unités de travail } \\
\text { agricole familiale }\end{array}$ & \multicolumn{2}{|c|}{1 à 2 utaf } & \multicolumn{2}{|c|}{2 à 4 utaf } & $\begin{array}{l}2 \text { à } 4 \text { utaf } \\
\text { ou > } 4 \text { utaf }\end{array}$ & $\begin{array}{l}1 \text { ou } 2 \text { utaf } \\
\text { (propriétaire) }\end{array}$ \\
\hline Types & $1 \mathrm{a}$ & $1 \mathrm{~b}$ & $2 a$ & $2 b$ & 3 & 4 \\
\hline Irrigation & \multicolumn{2}{|c|}{$\begin{array}{l}\text { location d'instrument } \\
\text { d'exhaure }\end{array}$} & \multicolumn{2}{|c|}{$\begin{array}{c}\text { copropriété ou location } \\
\text { de sakkia }\end{array}$} & $\begin{array}{l}\text { propriété } \\
\text { sakkia, pompe } \\
\text { (\& loueur) }\end{array}$ & $\begin{array}{l}\text { propriété } \\
\text { pompes }\end{array}$ \\
\hline Outillage & houe & araire & \multicolumn{2}{|c|}{$\begin{array}{c}\text { houe, araire } \\
\& \text { location de tracteur }\end{array}$} & $\begin{array}{c}\text { propriété } \\
\text { tracteurs } \\
\text { \& équipements }\end{array}$ & $\begin{array}{l}\text { propriété } \\
\text { tracteurs } \\
\text { et installations }\end{array}$ \\
\hline Elevage & absent & 1 u.a. & absent & $\begin{array}{l}\text { chargement } \\
\text { fort }\end{array}$ & $\begin{array}{l}\text { chargement } \\
\text { faible }\end{array}$ & $\begin{array}{l}\text { chargement } \\
\text { faible }\end{array}$ \\
\hline $\begin{array}{l}\text { Cultures } \\
\text { dominantes } \\
\text { en particulier }\end{array}$ & \multicolumn{4}{|c|}{$\begin{array}{c}\text { assolement collectif } \\
\text { avec }\end{array}$} & \multicolumn{2}{|c|}{$\begin{array}{c}\text { assolement } \\
\text { en partie libre } \\
\text { avec maraichage, vergers... }\end{array}$} \\
\hline \multirow[t]{2}{*}{ Culture } & \multicolumn{4}{|c|}{ familiale } & \multicolumn{2}{|c|}{ familiale \& salariale } \\
\hline & manuelle & attelée & manuelle & attelée & mécanisée & très mécanisée \\
\hline $\begin{array}{l}\text { Objectif } \\
\text { court terme }\end{array}$ & $\begin{array}{l}\text { gagner } \\
\text { argent }\end{array}$ & $\begin{array}{l}\text { garder } \\
\text { animaux }\end{array}$ & $\begin{array}{l}\text { gagner } \\
\text { argent }\end{array}$ & $\begin{array}{l}\text { garder } \\
\text { animaux }\end{array}$ & $\begin{array}{l}\text { maximiser } \\
\text { revenus }\end{array}$ & $\begin{array}{l}\text { maximiser } \\
\text { profit }\end{array}$ \\
\hline Stratégie & \multicolumn{2}{|c|}{ trouver travaux journaliers } & \multicolumn{2}{|c|}{ conserver le patrimoine } & $\begin{array}{l}\text { conserver } \\
\text { le patrimoine }\end{array}$ & $\begin{array}{c}s^{\prime} \text { ériger } \\
\text { en entreprises }\end{array}$ \\
\hline $\begin{array}{l}\text { Conditions } \\
\text { reproduction } \\
\text { sociale }\end{array}$ & \multicolumn{2}{|c|}{$\begin{array}{c}\text { maintien d'emplois } \\
\text { chez les autres exploitants } \\
\text { agricoles }\end{array}$} & \multicolumn{2}{|c|}{$\begin{array}{l}\text { affectation forces de travail } \\
\text { entre activités agricoles } \\
\text { et artisanales, migrations... }\end{array}$} & $\begin{array}{l}\text { scénarios } \\
\text { familiaux }\end{array}$ & $\begin{array}{c}\text { désengagement } \\
\text { de l'Etat }\end{array}$ \\
\hline $\begin{array}{l}\text { Représentativité/ } \\
\text { pop. agricole }\end{array}$ & \multicolumn{2}{|c|}{$50 \%$ familles } & \multicolumn{2}{|c|}{$40 \%$ familles } & $5 \%$ familles & $5 \%$ familles \\
\hline Superficie & \multicolumn{2}{|c|}{$15 \%$ surfaces } & \multicolumn{2}{|c|}{$35 \%$ surfaces } & $20 \%$ surfaces & $30 \%$ surfaces \\
\hline
\end{tabular}


d'engrais chimiques, très ancien en Egypte, et de produits phytosanitaires mal dosés, ajoutent aux problèmes d'épuration des eaux usées urbaines et provoquent des pollutions dommageables à la santé publique. La bilharziose est aussi la maladie la plus commune du pays, revers caché de la réussite apparente de l'agriculture.

\section{EETROUVER LES BASES D'UNE GESTION DURABLE DES TERRES ET DES EAUX}

L'Etat impose en Egypte une alternance de distribution de l'eau dans les biefs des canaux de 6 jours en eau et de 12 jours secs. L'usage des pompes diesels mobiles entraîne d'énormes problèmes pour l'administration hydraulique puisque les biefs des canaux sont parfois entièrement vidés, tandis que le système des sakkias écopait l'eau jusqu'à un niveau précis. Deux voies d'adaptation des institutions publiques de l'eau et des groupements d'agriculteurs existent.

La première, très technologique dans son approche, consiste à rendre l'eau encore plus rare par des aménagements hydrauliques étatiques qui retireraient aux fellahs la fonction d'exhaure. Sans revenir au schéma qui prévoyait d'irriguer tout le delta du Nil sans recours à aucun instrument de pompage, l'Etat peut installer des stations de moyenne puissance et restructurer les mailles hydrauliques avec une circulation permanente mais limitée de l'eau d'irrigation. A terme, cette évolution l'autoriserait à prélever soit une redevance hydraulique par hectare irrigué, soit une facturation par volume distribué. Cependant, des expériences similaires dans le monde ont montré une certaine difficulté de maintien à long terme des dispositifs de mesure et de paiement. En outre, toute la culture hydraulique égyptienne est fondée encore sur l'accès gratuit à l'eau du Nil, d'abord avec la crue, puis à l'échelle des fellahs, avec la maîtrise des sakkias et des pompes mobiles (qui n'est pas gratuite)

La seconde voie, plus institutionnelle, consisterait à rendre l'eau régulièrement et équitablement répartie par des accords formels entre le service des irrigations et les groupements de fellahs au sein du maillage hydraulique existant. Sans revenir à la sakkia contemporaine mue par des animaux, les fellahs ne souhaitant plus détourner leurs animaux des fonctions de production, un système de motorisation autonome de la sakkia pourrait constituer une base de progrès très intéressante pour l'ensemble des parties prenantes de la gestion de l'eau. L'Etat retrouverait un système cohérent de pompage régulier dans les biefs. Les paysans gagneraient à ne pas utiliser leurs animaux pour la traction mais plutôt pour la production laitière. La solidarité autour de l'instrument d'exhaure est aussi un facteur de stabilisation et permet d'éviter des conflits entre villages et mailles hydrauliques intravillageoises. Bien entendu, une telle évolution supposerait une concertation entre toutes les parties prenantes puisque la pratique du pompage complet du bief au moyen des pompes mobiles devrait être proscrite.

\section{VII}

L'élevage est la clé de voûte du système de production paysan par les trois fonctions essentielles au développement durable de l'agriculture irriguée: la force de traction, l'épargne sur pied, et la fertilisation organique. La sakkia a constitué une interface extraordinaire entre l'aménagement étatique et l'agriculture paysanne. Sa disparition répond surtout aux contraintes de l'alimentation fourragère des animaux, au moment où le marché des petites pompes mobiles s'est développé, avec des coûts d'exploitation assez faibles (énergie subventionnée). Mais les processus de gestion individuelle de l'eau posent des problèmes de cohérence d'ensemble aux différentes échelles des canaux d'irrigation.

L'Egypte, qui a su trouver au fil des deux derniers siècles un équilibre certainement efficace malgré les vicissitudes du drainage entre l'Etat aménageur et la multitude des fellahs producteurs de produits alimentaires frais et abondants, risque, au nom d'une politique libérale, de déstabiliser à la fois les paysans et les services chargés de rapprocher l'eau de leurs champs.

\section{BIBLIOGRAPHIE}

[1] Ayeb H. (1990). - La nécessaire révolution hydraulique en Egypte. Revue Tiers Monde, Tome XXXI, n 121, 29-56.

[2] Bakre M., Bethemont J., Commere R., Vant A. (1980). - L'Egypte et le Haut Barrage d'Assouan. Presses univ. de St-Etienne.

[3] BESANÇON J. (1957). - L'homme et le Nil. NRF Gallimard, Paris.

[4] CRaig G.M. (ed) (1993). - The Agriculture of Egypt. Oxford science publications.

[5] FanChette S. (1990). - Densités de population et urbanisation de l'espace rural : le cas du delta du Nil. Revue Tiers Monde, Tome XXXI, n' 21, 29-56.

[6] HuRst H.E. (1954). - Le Nil, description générale du fleuve, utilisation de ses eaux. Payot, Paris.

17] Molman A. (1956). - Machines à élever l'eau pour l'irrigation. FAO, col. progrès et mise en valeur, cah. 60 .

[8] RÉGNier Ph. (1989). - Les Saint-Simoniens en Egypte. Banque de l'U.E., Le Caire.

[9] Rivlin H. (1961). - The agricultural policy of Mohammed Ali in Egypt. Cambridge.

[10] RuF T. (1988), - Histoire contemporaine de l'agriculture égyptienne, essai de synthèse. ORSTOM, Etudes et thèses, Paris.

[11] RuF T. (1993), - The history of agricultural development In : Craig G.M. (ed), 1993. The Agriculture of Egypt. Oxford science publications, 188-208.

[12] RuF T. (1995). - Histoire hydraulique et agricole et lutte contre la salinisation dans le delta du Nil. Sécheresse $n^{\circ} 4$, vol. $6,307-317$. 